\title{
BMJ Open Risk factors for severity on admission and the disease progression during hospitalisation in a large cohort of patients with COVID-19 in Japan
}

\author{
Mari Terada, ${ }^{1,2}$ Hiroshi Ohtsu (D) , ${ }^{2}$ Sho Saito (D) , ${ }^{1}$ Kayoko Hayakawa, ${ }^{1,3}$ \\ Shinya Tsuzuki (D) , ,3 Yusuke Asai, ${ }^{3}$ Nobuaki Matsunaga, ${ }^{3}$ Satoshi Kutsuna, ${ }^{1}$ \\ Wataru Sugiura, ${ }^{2}$ Norio Ohmagari ${ }^{1,3}$
}

To cite: Terada M, Ohtsu H, Saito $\mathrm{S}$, et al. Risk factors for severity on admission and the disease progression during hospitalisation in a large cohort of patients with COVID-19 in Japan. BMJ Open 2021;11:e047007. doi:10.1136/ bmjopen-2020-047007

- Prepublication history and additional supplemental material for this paper are available online. To view these files, please visit the journal online. To view these files, please visit the journal online (http://dx.doi org/10.1136/bmjopen-2020047007).

Received 18 November 2020 Accepted 28 May 2021

Check for updates

(C) Author(s) (or their employer(s)) 2021. Re-use permitted under CC BY-NC. No commercial re-use. See rights and permissions. Published by BMJ.

${ }^{1}$ Department of Infectious Diseases, Disease Control and Prevention Center, National Center for Global Health and Medicine, Tokyo, Japan

${ }^{2}$ Center for Clinical Sciences, National Center for Global Health and Medicine, Tokyo, Japan ${ }^{3}$ AMR Clinical Reference Center, National Center for Global Health and Medicine, Tokyo, Japan

${ }^{4}$ Faculty of Medicine and Health Sciences, University of Antwerp, Antwerp, Belgium

Correspondence to

Dr Sho Saito;

ssaito@hosp.ncgm.go.jp

\section{ABSTRACT}

Objectives To investigate the risk factors contributing to severity on admission. Additionally, risk factors of worst severity and fatality were studied. Moreover, factors were compared based on three points: early severity, worst severity and fatality.

Design An observational cohort study using data entered in a Japan nationwide COVID-19 inpatient registry, COVIREGI-JP.

Setting As of 28 September 2020, 10480 cases from 802 facilities have been registered. Participating facilities cover a wide range of hospitals where patients with COVID-19 are admitted in Japan.

Participants Participants who had a positive test result on any applicable SARS-CoV-2 diagnostic tests were admitted to participating healthcare facilities. A total of 3829 cases were identified from 16 January to 31 May 2020, of which 3376 cases were included in this study.

Primary and secondary outcome measures Primary outcome was severe or nonsevere on admission, determined by the requirement of mechanical ventilation or oxygen therapy, Sp02 or respiratory rate. Secondary outcome was the worst severity during hospitalisation, judged by the requirement of oxygen and/orinvasive mechanical ventilation/extracorporeal membrane oxygenation.

Results Risk factors for severity on admission were older age, men, cardiovascular disease, chronic respiratory disease, diabetes, obesity and hypertension. Cerebrovascular disease, liver disease, renal disease or dialysis, solid tumour and hyperlipidaemia did not influence severity on admission; however, it influenced worst severity. Fatality rates for obesity, hypertension and hyperlipidaemia were relatively lower.

Conclusions This study segregated the comorbidities influencing severity and death. It is possible that risk factors for severity on admission, worst severity and fatality are not consistent and may be propelled by different factors. Specifically, while hypertension, hyperlipidaemia and obesity had major effect on worst severity, their impact was mild on fatality in the Japanese population. Some studies contradict our results; therefore, detailed analyses, considering in-hospital treatments, are needed for validation.

\section{Strengths and limitations of this study}

- This study investigated the disease progression of COVID-19, by comparing the risk factors on three points: early severity, worst severity throughout hospitalisation and fatality, whereas previous studies have predominantly reported worst severity.

- Categorisation used for worst severity may differ from those used in other studies as most cases in our data set did not include lung infiltration rate judged from radiological examination, $\mathrm{SpO}_{2}: \mathrm{FiO}_{2}$ ratio or $\mathrm{PaO}_{2}: \mathrm{FiO}_{2}$ ratio.

- The data set was derived from a large COVID-19 patient registry in Japan, which involves 299 facilities in Japan, which is both a strength and a limitation, as treatment methods and severity may vary.

- As treatment type, dosage, duration and combination varied immensely across the facilities, we did not consider treatments prior to and during hospitalisation.

Trial registration number UMIN000039873. https:// upload.umin.ac.jp/cgi-open-bin/ctr_e/ctr_view.cgi? recptno=R000045453

\section{INTRODUCTION}

COVID-19, caused by SARS-CoV-2, has caused a major global public health crisis. As of 3 October 2020, >34 million people had been infected in over 230 countries. ${ }^{12}$ Japan experienced two pandemic waves after the first case reported on 16 January 2020. During the first wave, a state of emergency was declared on 7th April, which ended on 25th May, settling the first wave. Nearly thrice as many SARS-CoV-2-positive cases were detected in the second wave, which emerged from the end of June. ${ }^{3}$ The fatality rate in the second wave has generally been lower in many countries, including Japan. ${ }^{4}$ 
When the number of patients explodes, hospital beds were in great shortage; hotels were used as isolation facilities in many countries. ${ }^{5-7}$ Likewise, in Japan, mild patients were transferred to hotels from April 2020. ${ }^{8}$ About two-thirds of cases did not require oxygen support throughout their illness. ${ }^{9}$ However, some cases initiated nonsevere may instantly plunge into a serious state and require aggressive care. ${ }^{10}$ Therefore, public health centres are in demand for indicators to identify those at a higher risk of aggravation in the early phase and determine the destination-hospital, hotel or home. Depicting the clinical course-from onset to worst severity and the outcome-is imperative to appropriately allocate patients to healthcare resources. Analyses considering the severity on admission and the disease progression, thereafter, has not been conducted are of interest to physicians globally.

We obtained nationwide data from a COVID-19 inpatient registry, 'COVID-19 REGISTRY JAPAN (COVIREGI-JP) ' and conducted a study to identify the independent risk factors contributing towards severity on admission. We aimed to determine the risk factors on admission, namely, demographics and comorbidities. Progression of severity was inspected in detail at different time points. Cases identified within the period of the first pandemic wave were studied.

\section{METHODS}

\section{Study design and patients}

This is an observational cohort study that uses the data accumulated in the nationwide 'COVID-19 REGISTRY JAPAN (COVIREGI-JP)' . As of 28 September 2020, 10048 cases from 802 facilities have been registered. Participating facilities cover a wide range of hospitals where patients with COVID-19 are admitted in Japan. Enrolled cases satisfied two eligibility criteria: a positive test result for COVID-19 and being admitted to a healthcare facility. Registration started on 2 March 2020 and is ongoing, at present.

\section{Patient and public involvement}

No patient involved.

\section{Data collection and case report form}

Data were collected in a case report form (CRF) developed for COVIREGI-JP. This CRF includes modified information of the International Severe Acute Respiratory and Emerging Infection Consortium CRF on COVID$19 .{ }^{11}$ On modification, we elaborated on data collection, especially on treatments, comorbidities and symptoms. In addition, as of 26 October 2020, this CRF underwent revisions two times to update therapeutic options or definitions, as new evidence emerges. Study data were collected and managed using Research Electronic Data Capture (REDCap) electronic data capture tools, ${ }^{12}{ }^{13}$ hosted at the datacenter in National Center for Global Health and Medicine. Data were either recorded on a CRF hard copy or were entered directly into REDCap at each facility.

\section{Comorbidities}

Comorbidities were collected based on Charlson comorbidity index ${ }^{14} 15$ with modifications. Some comorbidities were combined as follows: cardiovascular disease (CVD) - myocardial infarction, congestive heart failure and peripheral vascular disease; chronic respiratory disease (CRD) - chronic obstructive pulmonary disease (COPD) and other chronic lung diseases; renal disease or dialysis-moderate to severe renal disorder (creatinine $\geq 3 \mathrm{mg} / \mathrm{dL}$, nephropathy, postrenal transplantation or on dialysis) and maintenance haemodialysis or peritoneal dialysis before hospitalisation and solid tumour-solid tumour with or without metastasis. Obesity was diagnosed based on physician's judgement, and body mass index (BMI) was not considered in this study.

\section{Drug administration prior to and during hospitalisation}

Steroids, chemotherapy and immunosuppressants administered prior to hospitalisation were collected as prehospitalisation treatments. Steroids included those equivalent to $20 \mathrm{mg}$ /day prednisolone for $\geq 1 \mathrm{month}$ and are not considered as immunosuppressants. Chemotherapy and immunosuppressants were applicable if administered 3 months prior to hospitalisation. Treatment during hospitalisation was studied on systemic steroids, favipiravir, ciclesonide, heparin and tocilizumab, due to the frequent use in Japan. Heparin use included those given for both prophylactic and treatment purposes.

\section{Dataset}

We defined the first wave period from 16 January to 31 May $2020,{ }^{16}$ and cases from the first wave were included in this analysis. Therefore, data extraction conditions were (1) cases admitted to healthcare facilities between 16 January and 31 May 2020 and (2) all CRF items completed on data set generation. The data set was generated and fixed on 2 September 2020.

\section{Definitions of severity \\ Severity on admission}

Severity on admission was converted into bivariate variables: severe and nonsevere. Cases met at least one of the following criteria were categorised as severe: (1) requiring invasive or non-invasive mechanical ventilation (IMV), (2) requiring supplemental oxygen, (3) $\mathrm{SpO}_{2}$ $\leq 94 \%$ in room air or (4) tachypnea with respiratory rate $\leq 24 \mathrm{bpm} .{ }^{17}$ Those who did not meet the aforementioned were classified as nonsevere.

\section{Worst severity}

The worst severity was grouped into three categories: no-oxygen, oxygen and IMV/extracorporeal membrane oxygenation (ECMO). The worst state during hospitalisation was adopted on categorisation, and each was defined as follows:

No-oxygen-no requirement of supplemental oxygen throughout hospitalisation. 
Oxygen-required supplemental oxygen (including high-flow oxygen devices) or non-IMV during hospitalisation.

IMV/ECMO-required IMV or ECMO during hospitalisation.

\section{Statistical analysis}

Continuous variables are presented in median and IQR and categorical variables in number of cases and percentages. We classified the disease progression into three stages: severity on admission, worst severity and clinical outcomes. We used Mann-Whitney U tests (for two groups) or Kruskal-Wallis tests (for three groups) for continuous variables and $\chi^{2}$ tests for categorical variables.

We conducted univariate analyses and a multivariable logistic regression analysis to identify the factors associated with the patients' severity on admission. We included age, sex, comorbidities (CVD, cerebrovascular disease, CRD, asthma, liver disease, diabetes, obesity diagnosed by physicians, renal disease or dialysis, solid tumours, leukaemia, lymphoma, hypertension and hyperlipidaemia), use of systemic steroids in the past month, chemotherapy in the past 3 months and use of immunosuppressants other than steroids as independent variables. As for univariate analysis, we conducted logistic regression analysis about days between onset and admission and age. As for multivariate analysis, multicollinearity was evaluated using the variance inflation factor (VIF). Variables of VIF $>3$ were excluded from the model; however, no variables demonstrated obvious multicollinearity. The variables included in the model were chosen based on the previous findings ${ }^{18-20}$ and expert opinions.

R V.3.6.3 (R core team, 2020) ${ }^{21}$ was used for all the analyses performed in this study.

\section{RESULTS}

Within the study period, 3829 cases were identified and 3376 cases from 299 facilities were included in this study. Of them, 2199 cases $(65.1 \%)$ were nonsevere, and 1181 cases $(34.9 \%)$ were severe at the time of admission. After categorising the two groups further into no-oxygen, oxygen and IMV/ECMO by worst severity, compositions were $1758(81.5 \%), 357(16.5 \%)$ and $43(2.0 \%)$ for the nonsevere group and 190 (16.1\%), $677(57.5 \%)$ and 311 $(26.4 \%)$ for the severe group, respectively. While categorising the cases, $44(1.3 \%)$ were unavailable due to missing values.

Demographics and clinical characteristics of the study population are shown in table 1. Days between onset and admission were similar in both groups (nonsevere 6.0 vs severe 7.0 days). Over 10 times as many severe cases on admission underwent IMV/ECMO than nonsevere cases $(2.0 \%$ vs $26.4 \%)$. Severe cases were older (50.0 vs 67.0$)$, had higher BMI (22.9 vs 24.1), greater male dominance $(56.3 \%$ vs $70.5 \%)$ and a higher prevalence of comorbidities excluding leukaemia, compared with the nonsevere group. The most prevalent symptoms in both groups were fever (nonsevere $49.5 \%$, severe $73.7 \%$ ), cough (nonsevere $53.8 \%$, severe $64.9 \%$ ) and fatigue (nonsevere $40.3 \%$, $59.9 \%$ ) but was greater in the severe group. Conversely, prevalence of dysgeusia (25.9\% vs $13.2 \%$ ), dysosmia (22.6\% vs $11.5 \%)$, headache $(18.1 \%$ vs $14.7 \%)$ and runny nose $(11.9 \%$ vs $8.9 \%)$ was higher in the nonsevere group.

Results of univariate analyses about factors associated with being severe cases on admission are described in table 2. In most variables, univariate analysis showed similar results compared with the multivariate analysis. Results of the multivariate logistic regression to determine the risk of severity on admission are shown in table 3. Older age (OR 1.04 (1.03-1.04)) and men (OR 2.09 (1.76-2.48)) were considered a risk among the demographics and comorbidities including CVD (OR 1.48 (1.04-2.10)), respiratory disease (OR 2.51 (1.67-3.78)), diabetes (OR 1.34 (1.09$1.64)$ ), obesity (OR $1.75(1.26-2.45)$ ) and hypertension (OR 1.33 (1.08-1.64)). Days between onset to admission were nonsignificant $(\mathrm{p}=0.960)$; the timing of admission did not affect the severity on admission. Cerebrovascular disease and hyperlipidaemia were not associated with the severity at admission after other confounding factors were considered, although they showed different results in univariate analyses.

Table 4 depicts the study population from a different angle and is categorised by the worst severity $(n=3336)$ and fatality $(n=3376)$. Oxygen and IMV/ECMO cases were predominantly severe at admission $(65.5 \%$ and $87.9 \%$, respectively), whereas most no-oxygen cases come from non-severe group (90.2\%). Prevalence of comorbidities was lowest in no-oxygen cases; however, prominent difference was not observed for asthma. Similarly, fatal cases were more severe at admission $(84.0 \%$ vs $31.1 \%)$ and had higher prevalence of oxygen and IMV/ECMO cases (oxygen: $56.4 \%$ vs $29.0 \%$, IMV/ECMO: $41.9 \%$ vs $8.2 \%$, respectively). Days between onset and admission were longer in nonfatal cases ( 5 days vs 7 days).

More nonsevere cases with any comorbidity underwent treatment with oxygen or IMV/ECMO compared with nonsevere cases with no comorbidities. In figure 1 , only $11.9 \%$ underwent oxygen therapy or IMV/ECMO in nonsevere cases without any comorbidities. However, among the nonsevere cases with comorbidity, the rates of oxygen or IMV/ECMO were higher in most comorbidities, including CVD (34.7\%), CRD (38.9\%), liver disease $(36.7 \%)$, diabetes $(35.1 \%)$, obesity $(35.8 \%)$, cerebrovascular disease $(34.7 \%)$, renal disease $(40.9 \%)$, solid tumour $(27.3 \%)$, hypertension $(31.2 \%)$ and hyperlipidaemia $(25.0 \%)$. Asthma alone followed a different trend; the chances of oxygen and IMV/ECMO requirement was lower.

Among the cases without comorbidity, $75.2 \%$ of cases that were severe on admission required oxygen or IMV/ ECMO; however, the fatality rate was low, and only $8.0 \%$ resulted in death (figure 2). Fatality rates were approximately 3-five times higher when the following comorbidities were present: renal disease or dialysis (44\%), CVD $(40.5 \%)$, cerebrovascular disease $(39.5 \%)$, CRD (30.4\%), 
Open access

Table 1 Characteristics of patients included in the present study

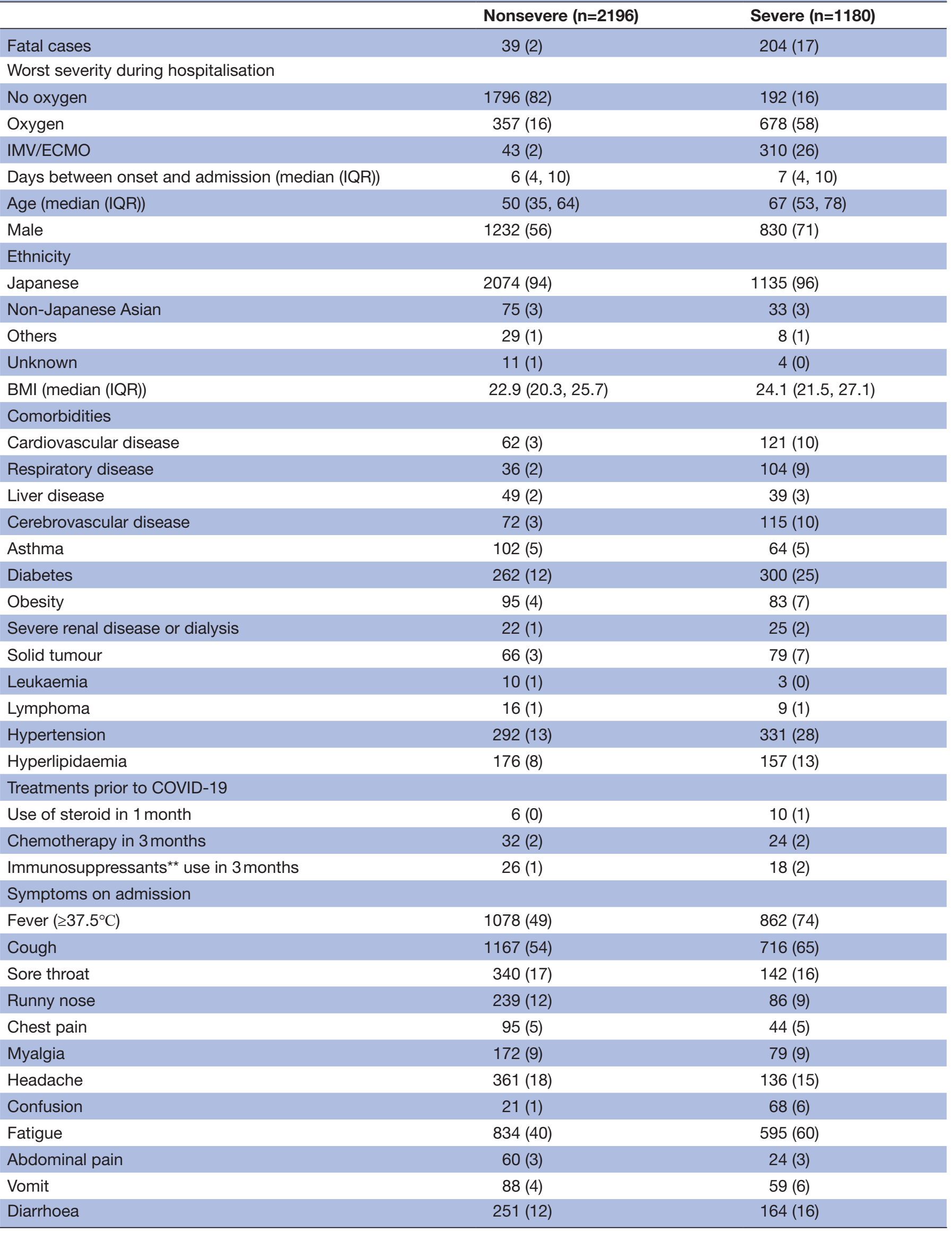

Continued 
Table 1 Continued

\begin{tabular}{lcr}
\hline & Nonsevere $(\mathbf{n}=\mathbf{2 1 9 6})$ & Severe $(\mathbf{n}=\mathbf{1 1 8 0})$ \\
\hline Dysgeusia & $494(26)$ & $113(13)$ \\
Dysosmia & $422(23)$ & $96(12)$ \\
\hline
\end{tabular}

*Immunosupprenssants other than steroids.

$\mathrm{BMI}$, body mass index; IMV/ECMO, invasive mechanical ventilation/extracorporeal membrane oxygenation.

solid tumour (30.4\%), diabetes (25.8\%), and liver disease $(25.6 \%)$. Even among non-severe cases, relatively high fatality rate was observed in cases with solid tumour, CRD, cerebrovascular disease, CVD, and renal disease or dialysis, with fatality rates ranging from $8.1 \%$ to $11.1 \%$. Collectively, obesity, hypertension and hyperlipidaemia influenced the worst severity; however, their influence on fatality was relatively lower than that mentioned earlier.

Older age was relevant to both worst severity and fatality, as shown in online supplemental figures 12 . The combined proportion of oxygen and IMV/ECMO increased gradually by age from $5.3 \%$ in 20 s to $69.3 \%$ in $\geq 80 \mathrm{~s}$. Conversely, the fatality rate leaped between $60 \mathrm{~s}$ $(2.2 \%)$ and $70 \mathrm{~s}(8.6 \%)$. Likewise, online supplemental figure 3 shows the combined proportion of oxygen and IMV/ECMO and fatality rates as higher in older individuals, irrespective of underlying comorbidities.
Predominant comorbid cases required more drug administration than those without comorbidities (online supplemental table 1). Systemic steroids were most frequently used in cases with CRD (27.9\%). Heparin was used most often in renal disease $(12.8 \%)$, hypertension (11.2\%), diabetes $(10.9 \%)$ and CVD (10.4\%).

\section{DISCUSSION}

We took disease progression into consideration and evaluated the study population based on severity on admission, worst severity and the outcome. To our knowledge, studies have predominantly reported worst severity, whereas disease progression has not been considered. Our findings, therefore, are novel, augmenting the evidence needed to depict the clinical course and trajectory from onset to worsening condition. Specifically, this

Table 2 Factors associated with being 'severe' at the time of admission (univariate analysis)

\begin{tabular}{|c|c|c|c|}
\hline & OR & $95 \% \mathrm{CI}$ & $P$ value \\
\hline Days between onset and admission & 1.0 & 0.99 to 1.01 & 0.897 \\
\hline Age & 1.04 & 1.04 to 1.05 & $<0.001$ \\
\hline Male & 1.85 & 1.59 to 2.16 & $<0.001$ \\
\hline \multicolumn{4}{|l|}{ Comorbidities } \\
\hline Cardiovascular disease & 3.93 & 2.84 to 5.48 & $<0.001$ \\
\hline Cerebrovascular disease & 3.18 & 2.33 to 4.38 & $<0.001$ \\
\hline Chronic respiratory disease & 5.80 & 3.90 to 8.78 & $<0.001$ \\
\hline Asthma & 1.18 & 0.84 to 1.64 & 0.318 \\
\hline Liver disease & 1.50 & 0.95 to 2.34 & 0.070 \\
\hline Diabetes & 2.52 & 2.08 to 3.04 & $<0.001$ \\
\hline Obesity diagnosed by physicians & 1.67 & 1.22 to 2.29 & 0.001 \\
\hline Severe renal disease or dialysis & 2.14 & 1.15 to 4.00 & 0.013 \\
\hline Solid tumour & 2.32 & 1.63 to 3.29 & $<0.001$ \\
\hline Leukaemia & 0.56 & 0.10 to 2.17 & 0.562 \\
\hline Lymphoma & 1.05 & 0.41 to 2.53 & 0.999 \\
\hline Hypertension & 2.54 & 2.12 to 3.05 & $<0.001$ \\
\hline Hyperlipidaemia & 1.76 & 1.39 to 2.23 & $<0.001$ \\
\hline \multicolumn{4}{|l|}{ Treatments prior to COVID-19 } \\
\hline Use of steroid in 1 month & 3.12 & 1.02 to 10.47 & 0.032 \\
\hline Chemotherapy in 3 months & 1.40 & 0.79 to 2.47 & 0.258 \\
\hline Immunosuppressants* use in 3 months & 1.29 & 0.67 to 2.46 & 0.428 \\
\hline
\end{tabular}

*Immunosupprenssants other than steroids. 
Table 3 Factors associated with being 'severe' at the time of admission

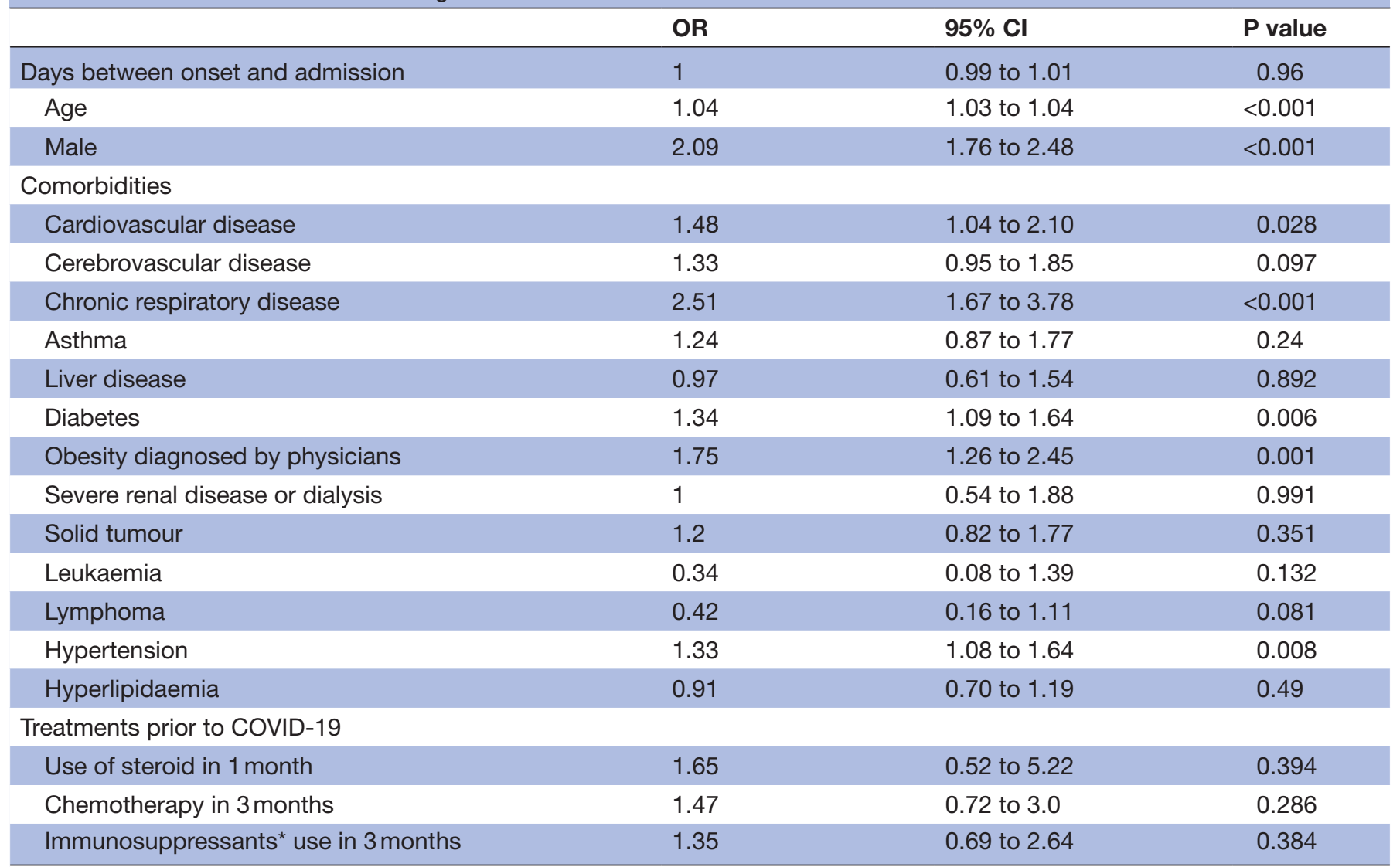

*Immunosupprenssants other than steroids.

study segregated the comorbidities influencing severity and death. Based on our findings, it may be possible that the early severity, worst severity and death are propelled by different factors, while confirmation is necessary by multivariate analysis.

The majority of comorbidities we studied did not influence severity on admission. On admission, severity was driven by age, sex, CVD, CRD, diabetes, obesity and hypertension. The trend was similar for the worst severity, as cases with these factors had higher rate of oxygen or IMV/ECMO. However, all comorbidities appeared to influence the worst severity.

Within the comorbidities, the prognosis of cases with obesity, hypertension or hyperlipidaemia was relatively favourable. In contrast to our results, hypertension and obesity are reportedly related to an increased risk of severity and mortality. ${ }^{2-25} \mathrm{~A}$ large cohort reported a trend similar to our results, ${ }^{18}$ whereas other studies reported that obesity is being confounded by age and sex. ${ }^{26}{ }^{27}$ The presence of hypertension and the use of angiotensinconverting enzyme (ACE) inhibitors and angiotensin II receptor blockers act contrarily, ${ }^{28-30}$ while ACE2 mediates the entry of SARS-CoV-2 into host cells ${ }^{31}{ }^{32}$; thus, COVID-19 pathophysiology in hypertensive patients becomes intricate.

In accordance with previous studies, CVD, CRD, liver disease, diabetes, cerebrovascular disease, renal disease or dialysis and solid tumour were associated with fatality and worst severity. Two meta-analyses have reported common risk factors for worst severity during hospitalisation, which include diabetes, COPD, malignancy, CVD and cerebrovascular disease. ${ }^{33}$ Other studies have also reported chronic liver disease and renal disease as risk factors. ${ }^{35-37}$ Studies have elucidated that acute respiratory distress syndrome and coagulation dysfunction are related to the renin-angiotensin-aldosterone system and blood coagulation pathways, which are altered by SARS-CoV-2 host cell invasion via ACE2. ${ }^{38-40}$ Clinical and nonclinical studies revealed an association between these comorbidities, while SARS-CoV-2 infection decreases ACE2 expression, ACE2 deficiency is reported to cause cardiac overload and kidney inflammation. ${ }^{40-43}$ Elevated blood glucose is also associated with mortality. ${ }^{44}$ Although risk factors vary among studies, the comorbidities we identified are highly likely associated with fatality, backed up by clinical and nonclinical results.

Different trends were seen in the rates of IMV/ECMO and death for each comorbidity. Although rates of IMV/ ECMO were comparable in all comorbid cases, those with obesity, asthma, hyperlipidaemia and hypertension showed a lower fatality rate, suggesting that the fatality rates within the IMV/ECMO cases with these comorbidities were lower than expected. Contrarily, fatality rates in cases with CVD, cerebrovascular disease, renal 
Table 4 Characteristics of patients stratified by non-fatal/fatal cases and severity during hospitalisation

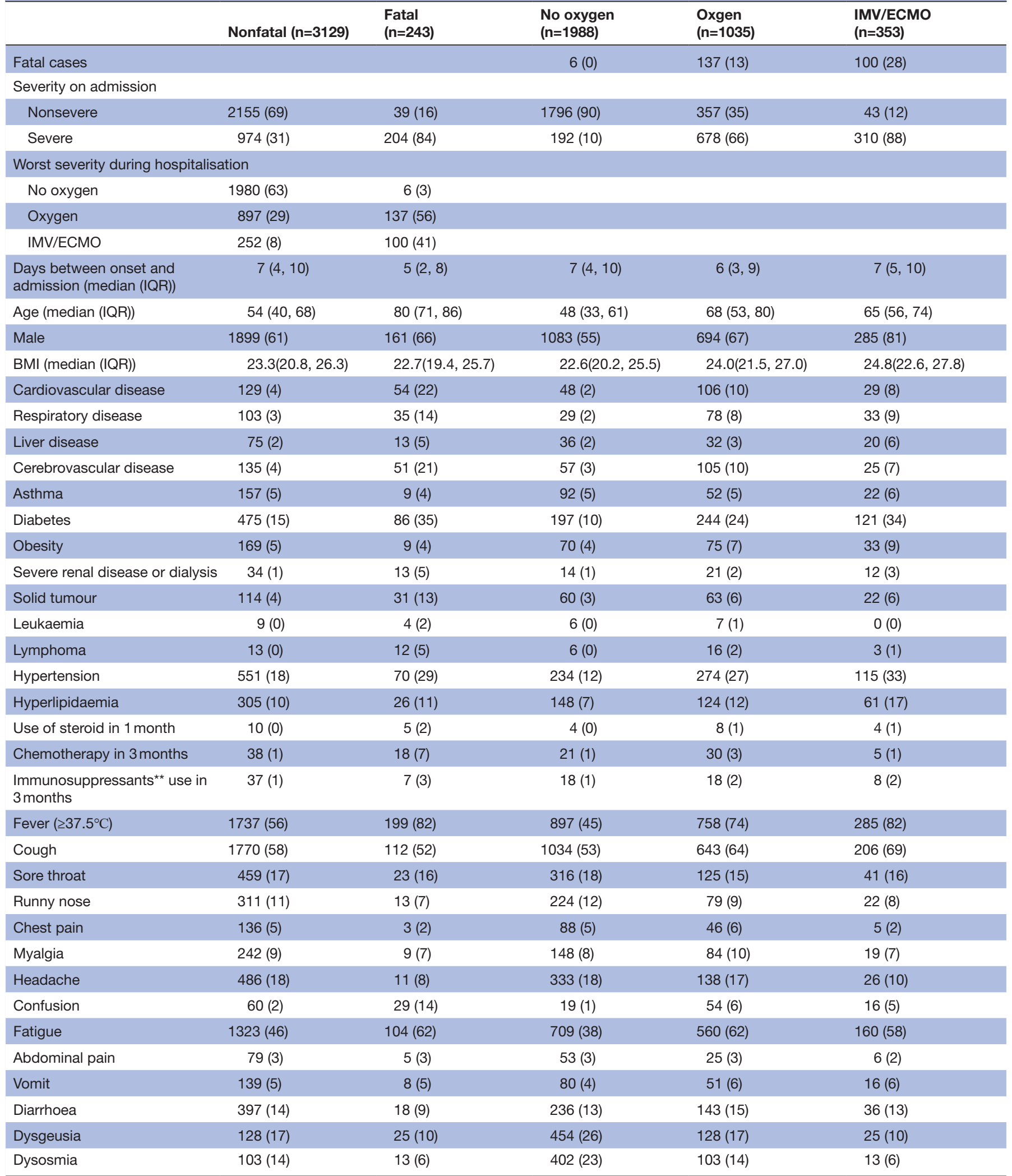

*Immunosupprenssants other than steroids.

IMV/ECMO, invasive mechanical ventilation/extracorporeal membrane oxygenation. 

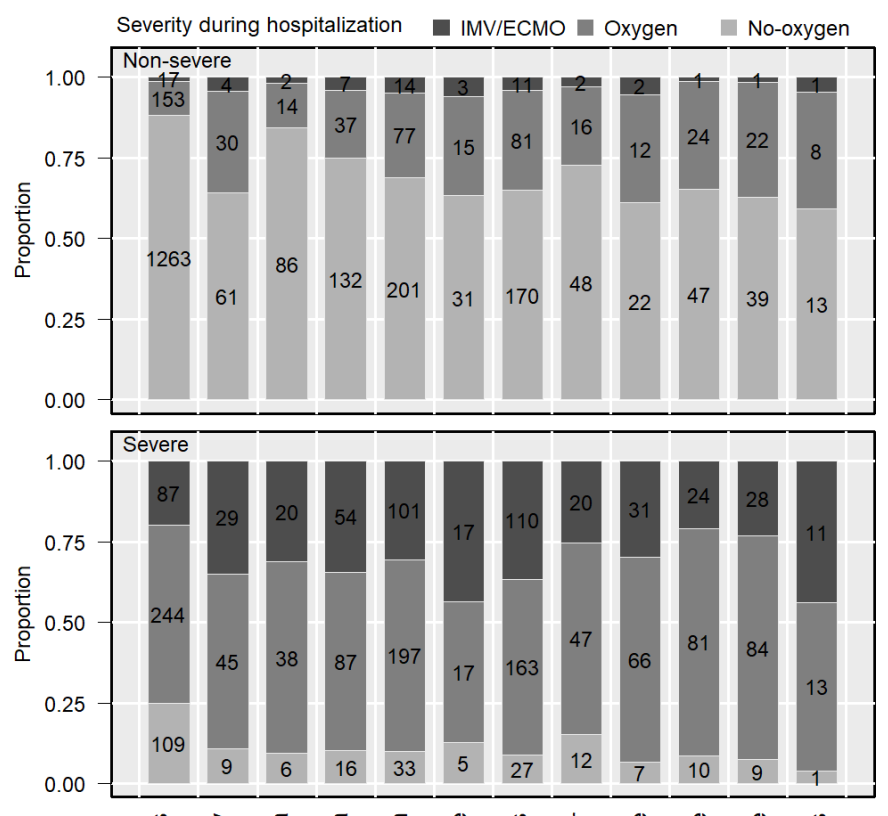

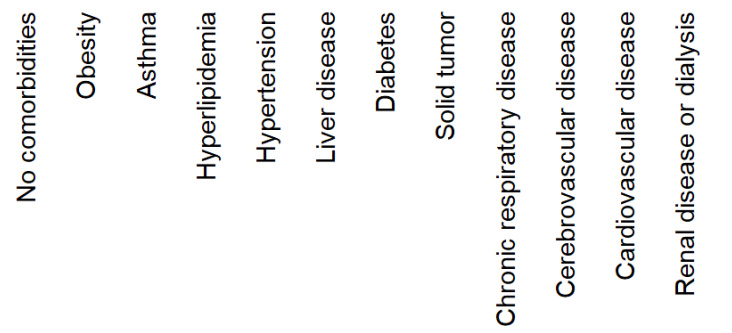

Figure 1 Distribution of the worst severity arranged by severe/nonsevere at admission and presence/absence of comorbidities. Top bars represent nonsevere cases at admission and bottom bars represent severe cases at admission. Each group of cases was divided based on the presence of comorbidities. Bars represent different categories of worst severity: light grey-no-oxygen, darker grey-oxygen and darkest grey-IMV/ECMO. IMV/ECMO, invasivemechanical ventilation/extracorporeal membrane oxygenation.

dysfunction, tumour and CRD were comparable or higher than IMV/ECMO rates. The number of death actually exceeded the number of IMV/ECMO cases in patients with tumour, cerebrovascular disease or CVD. These comorbidities likely have caused a higher risk of death and some even died without intubation. Healthcare nearly overwhelmed in the first wave in Japan, but ICU capacity was maintained, ${ }^{45}$ and, thus, intubation may have been unperformed due to a medical judgement. A detailed examination of these issues is necessary in the future.

Our results did not show prominent difference in fatality between men and women. Oftentimes, men are considered to develop severe conditions and increased fatality. ${ }^{36} 3746-48$ However, according to Global Health 5050 , sexual disparity in incidence of COVID-19 is low. ${ }^{49}$ Additionally, ACE2 expression is affected by sexual hormones, whereby higher expression is observed in men, possibly explaining the sexual disparity ${ }^{50-52}$ Moreover, the immunological response to produce antibodies
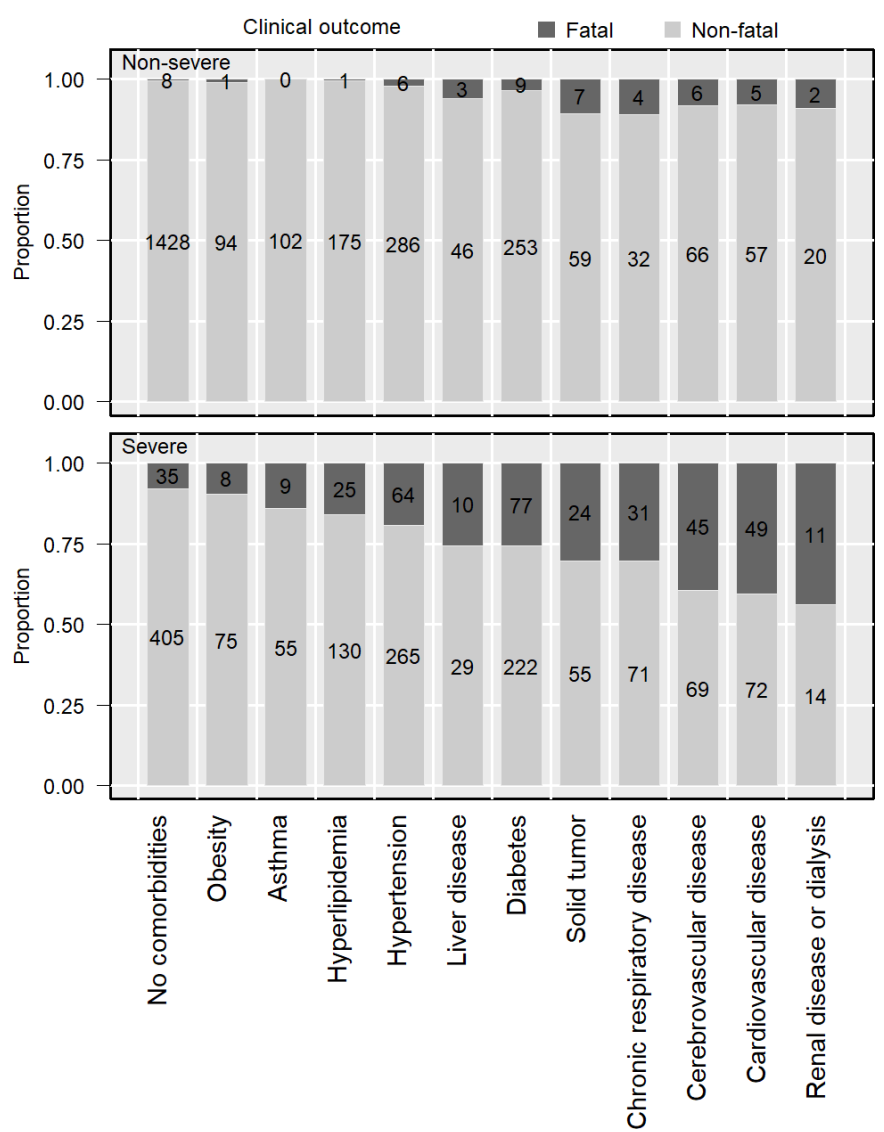

Figure 2 Distribution of the fatality arranged by severe/ nonsevere at admission and presence/absence of comorbidities. Top bars represent nonsevere cases at admission and bottom bars represent severe cases at admission. Each group of cases was divided based on the presence of comorbidities. Dark grey represents fatal cases while light grey represents nonfatal cases.

is more favourable in women. ${ }^{53}$ These studies support the rationale that men are more susceptible to severe COVID-19, which contravene our results. The lowerthan-expected fatality rate in our male population may be attributed to comorbidity prevalence, treatments, age and/or degree of obesity.

Fatality rates were comparable between asthmatic and cases without comorbidities in our results. Theoretically, COVID-19 can be a risk for patients with asthma. A viral respiratory infection is presented as relatively worse and causes asthma exacerbation. ${ }^{54}$ Asthmatic patients reportedly require a longer duration of mechanical ventilation when intubated ${ }^{56}$; however, no study, including ours, has found strong evidence on severity or mortality. ${ }^{57-60}$ Inhaled corticosteroids (ICS) are known to downregulate $\mathrm{ACE} 2^{6162}$ and are being investigated for treating COVID-19. ${ }^{63}$ ICS may have impeded aggravation in patients with asthma with COVID-19. ${ }^{64}$ Overall, further studies are needed to elucidate the true risk of asthma on COVID-19.

The variability in the risk factors may be explained by the differences in study population, definition for comorbidities and ethnicity. First, the rate of comorbid 
patients had been lower in our cohort as suggested by extensive cohort studies. ${ }^{19} 2065$ The degree of obesity may also have been milder, as the average BMI is lower in the Asia-Pacific region than in other global regions. ${ }^{66}$ Second, obesity was judged by a physician in our study, and the results may change if BMI was incorporated. Ethnic differences due to genetic properties are also plausible. Individuals with stronger binding affinities of human leucocyte antigen (HLA) proteins to SARS-CoV-2 virus peptides are less likely become severe or fatal, ${ }^{67-70}$ and ethnic differences are present in HLA allele frequency. ${ }^{71} \mathrm{~A}$ few strong binder alleles were more frequent in Northeast Asians; however, the complete picture is complicated. ACE1 polymorphism ${ }^{72}$ and Neanderthal haplotype ${ }^{73}$ were also suggestive of lower risk of COVID-19 among Asians and East Asians, respectively. Additionally, ethnic differences other than genetic traits are also anticipated. Vitamin D deficiency is postulated to increase COVID-19 severity, whereas vitamin D deficiency is correlated to Northern latitude. ${ }^{74}$ Within the elderly population, higher rates of deficiencies were observed in North America and Europe compared with Japan. ${ }^{75}$ Although our study did not examine vitamin $\mathrm{D}$, these facts also allow us to expect lower severity and fatality in Japan.

The period of when the COVID-19 occurred, and the situation of pandemic and healthcare provision should also be noted when discussing severity and fatality. The longer our struggle against COVID-19 pandemic becomes, the more complicated interpretation be required due to chronological, regional and viral transition. In the two pandemic waves of COVID-19 in Japan, the patient population altered; median age, rates of comorbidities and fatality rate had become smaller in the second than the first. ${ }^{76}$ Similar trend was observed in other countries. ${ }^{77} 78$ These differences might be explained, at least partially, by the timing of drug approval for remdesivir (approved in May 2020) and newly revealed efficacy of dexamethasone against COVID-19 in June. Our data set includes nationwide data during the first wave, and articles referred elsewhere for comparison included data from a period close to ours.

Our results could be useful to roughly identify those at a risk of aggravation or death. Days from onset to admission were not a risk factor; early hospitalisation will not influence the disease progression or outcome, and severity on admission was mostly driven by age and the presence of a few comorbidities. Several studies have created a scoring system to predict the risk of severity or mortality. ${ }^{79-81}$ However, these use laboratory data collected on admission and are seldom practical for estimating the severity of illness prior to medical visits or when test results are not promptly available. While these are useful to predict prognosis more precisely, our results are useful from a public health perspective, as they provide risk factors for predicting the severity on admission and disease progression from patients' background factors.

Our study has several limitations. Although the definition of severe and nonsevere was adopted from a previous study, ${ }^{17}$ such definition is not common as worst severity is used frequently or otherwise point of evaluation is unspecified. Our categorisation of worst severity also differs from other definitions. ${ }^{82} 83$ We did not adopt radiological criteria as lung infiltration rate was not collected in the registry where our data set was extracted. Ratio of arterial oxygen saturation or arterial partial oxygen pressure $(\mathrm{PaO} 2)$ to the fraction of inspired oxygen (FiO2) was not used as data were available for limited number of cases. This fact may have caused differences in risk factors. We did not consider treatments prior to and during hospitalisation nor did we incorporate laboratory test results in the analysis, which may be persisting as confounders. As our data were collected from hundreds of healthcare facilities, treatment type, dosage, duration and combination varied immensely; laboratory tests also varied as reporting units and standard reference ranges were different across facilities. Treatments may be confounding also in terms of drug approval, as explained elsewhere. Thorough data verification and analytical deliberation are required before usage of these data; thus, we did not include them in the current analysis. Moreover, hotels were used as isolation facilities from April 2020, and participant selection might have altered thereafter. COVIREGI-JP is continuously open for new entry; the number of registrations is increasing, and subsequent results may vary from ours.

\section{CONCLUSION}

On admission, factors that influence severity were age, sex and comorbidities, including CVD, CRD, diabetes, obesity and hypertension. Risk factors for severity on admission, worst severity and fatality were not consistent, and it is likely that they are each propelled by different factors. Our results are practically useful for predicting the progression and preparing for the worst, based on patients' backgrounds. Moreover, based on our predictions, healthcare resources can be allocated to patients in the most suitable way.

Acknowledgements We thank all the participating facilities for their care towards patients with COVID-19 and cooperation during data entry. We are especially grateful for the 299 facilities that contributed to the dataset used in this study.

Contributors MT conceived and $\mathrm{HO}, \mathrm{SS}, \mathrm{KH}, \mathrm{ST}$ and MT designed the study. ST, YA, SS, KH and MT analysed and interpreted the data. MT and ST drafted the first version of the manuscript. All the authors including NM, SK, SW, NO, and those stated above contributed to, read, and approved the final manuscript. The corresponding author attests that all listed authors meet authorship criteria and that no others meeting the criteria have been omitted. SS is the guarantor.

Funding This study was funded by Health and Labour Sciences Research Grant, 'Research for risk assessment and implementation of crisis management functions for emerging and re-emerging infectious diseases', provided by the Japanese Ministry of Health, Labour, and Welfare (grand number 19HA1003). The funding agency did not assume any role in this study or COVIREGI-JP. 
Competing interests $\mathrm{HO}$ reports personal fees as a statistician and as an external consultant for clinical trials from EPS International, outside the submitted work.

\section{Patient consent for publication Obtained.}

Ethics approval The National Center for Global Health and Medicine ethics board approved this study (referral number NCGM-G-003494-08) and waived the need for informed consent from individual patients owing to the non-invasive, noninterventional nature of this observational study according to the local Ethical Guidelines (https://www.mhlw.go.jp/file/06-Seisakujouhou-10600000-Daijinkanbou kouseikagakuka/0000080278.pdf). Information regarding opting out of our study is available on the COVIREGl-JP website (https://covid-registry.ncgm.go.jp/). Although it is not mandatory, the study is also being registered on trial registration website (https://upload.umin.ac.jp/cgi-open-bin/ctr_e/ctr_view.cgi?recptno=R000045453).

Provenance and peer review Not commissioned; externally peer reviewed.

Data availability statement Data are available upon reasonable request. Data on an individual level is shared with limitation to participating healthcare facilities through applications to COVIREGI-JP.

Supplemental material This content has been supplied by the author(s). It has not been vetted by BMJ Publishing Group Limited (BMJ) and may not have been peer-reviewed. Any opinions or recommendations discussed are solely those of the author(s) and are not endorsed by BMJ. BMJ disclaims all liability and responsibility arising from any reliance placed on the content. Where the content includes any translated material, BMJ does not warrant the accuracy and reliability of the translations (including but not limited to local regulations, clinical guidelines, terminology, drug names and drug dosages), and is not responsible for any error and/or omissions arising from translation and adaptation or otherwise.

Open access This is an open access article distributed in accordance with the Creative Commons Attribution Non Commercial (CC BY-NC 4.0) license, which permits others to distribute, remix, adapt, build upon this work non-commercially, and license their derivative works on different terms, provided the original work is properly cited, appropriate credit is given, any changes made indicated, and the use is non-commercial. See: http://creativecommons.org/licenses/by-nc/4.0/.

\section{ORCID iDs}

Hiroshi Ohtsu http://orcid.org/0000-0003-3261-8828

Sho Saito http://orcid.org/0000-0002-9981-7584

Shinya Tsuzuki http://orcid.org/0000-0002-8504-1244

\section{REFERENCES}

1 World Health Organization. Coronavirus disease (COVID-19) pandemic. Available: https://www.who.int/emergencies/diseases/ novel-coronavirus-2019 [Accessed 03 Oct 2020].

2 Huang C, Wang Y, Li X, et al. Clinical features of patients infected with 2019 novel coronavirus in Wuhan, China. Lancet 2020;395:497-506.

3 Our World in Data. Japan: coronavirus pandemic country profile. Available: https://ourworldindata.org/coronavirus/country/japan? country= JPN [Accessed 03 Oct 2020].

4 Fan G, Yang Z, Lin Q, et al. Decreased case fatality rate of COVID-19 in the second wave: a study in 53 countries or regions. Transbound Emerg Dis 2021;68:213-5

5 Bruni T, Lalvani A, Richeldi L. Telemedicine-enabled accelerated discharge of patients hospitalized with COVID-19 to isolation in repurposed hotel rooms. Am J Respir Crit Care Med 2020;202:508-10.

6 Fenton ME, Wasko K, Behl V, et al. An expanded COVID-19 telemedicine intermediate care model using repurposed hotel rooms. Am J Respir Crit Care Med 2020;202:1190-2.

7 Ramírez-Cervantes KL, Romero-Pardo V, Pérez-Tovar C, et al. A Medicalized hotel as a public health resource for the containment of Covid-19: more than a place for quarantining. J Public Health 2021;43:89-97.

8 Hayasaki E. Covid-19: how Japan squandered its early jump on the pandemic. BMJ 2020;369:m1625.

9 Matsunaga N, Hayakawa K, Terada M. Clinical epidemiology of hospitalized patients with COVID-19 in Japan: report of the COVID-19 registry Japan. Clin Infect Dis 2021:ciaa1470.

10 Duan J, Wang X, Chi J, et al. Correlation between the variables collected at admission and progression to severe cases during hospitalization among patients with COVID-19 in Chongqing. J Med Virol 2020;92:2616-22

11 International Severe Acute Respiratory and Emerging Infection Consortium ISARIC). COVID-19 clinical research resources.
Available: https://isaric.tghn.org/covid-19-clinical-researchresources/ [Accessed 03 Oct 2020].

12 Harris PA, Taylor R, Thielke R, et al. Research electronic data capture (REDCap)--a metadata-driven methodology and workflow process for providing translational research informatics support. J Biomed Inform 2009;42:377-81.

13 Harris PA, Taylor R, Minor BL, et al. The REDCap consortium: Building an international community of software platform partners. $J$ Biomed Inform 2019:95:103208.

14 Charlson ME, Pompei P, Ales KL, et al. A new method of classifying prognostic comorbidity in longitudinal studies: development and validation. J Chronic Dis 1987;40:373-83.

15 Schneeweiss S, Wang PS, Avorn J, et al. Improved comorbidity adjustment for predicting mortality in Medicare populations. Health Serv Res 2003;38:1103-20.

16 Ministry of Health, Labour, and Welfare. Fatality and severity risk factor of COVID-19 (article in Japanese). Available: https://www. mhlw.go.jp/content/10900000/000662183.pdf [Accessed 03 Oct 2020].

17 Beigel JH, Tomashek KM, Dodd LE, et al. Remdesivir for the Treatment of Covid-19 - Final Report. N Engl J Med 2020;383:1813-26.

18 Fried MW, Crawford JM, Mospan AR, et al. Patient characteristics and outcomes of 11721 patients with coronavirus disease 2019 (COVID-19) hospitalized across the United States. Clin Infect Dis 2021;72:e558-65.

19 Karagiannidis C, Mostert C, Hentschker C, et al. Case characteristics, resource use, and outcomes of 10021 patients with COVID-19 admitted to 920 German hospitals: an observational study. Lancet Respir Med 2020;8:853-62.

20 Kalyanaraman Marcello R, Dolle J, Grami S, et al. Characteristics and outcomes of COVID-19 patients in New York City's public hospital system. PLoS One 2020;15:e0243027.

21 R Core Team. R: a language and environment for statistical computing. Vienna, Austria: R Foundation for Statistical Computing, 2020. https://www.R-project.org/

22 Hussain A, Mahawar K, Xia Z, et al. Obesity and mortality of COVID-19. meta-analysis. Obes Res Clin Pract 2020;14:295-300.

23 de Siqueira JVV, Almeida LG, Zica BO. Impact of obesity on hospitalizations and mortality, due to COVID-19: a systematic review. Obes Res Clin Pract 2020;S1871-403X:30553-6.

24 Pranata R, Lim MA, Huang I, et al. Hypertension is associated with increased mortality and severity of disease in COVID-19 pneumonia: a systematic review, meta-analysis and meta-regression. J Renin Angiotensin Aldosterone Syst 2020;21:1470320320926899.

25 Lippi G, Wong J, Henry BM. Hypertension in patients with coronavirus disease 2019 (COVID-19): a pooled analysis. Pol Arch Intern Med 2020;130:304-9.

26 Tartof SY, Qian L, Hong V, et al. Obesity and mortality among patients diagnosed with COVID-19: results from an integrated health care organization. Ann Intern Med 2020;173:M20-3742.

27 Palaiodimos L, Kokkinidis DG, Li W, et al. Severe obesity, increasing age and male sex are independently associated with worse inhospital outcomes, and higher in-hospital mortality, in a cohort of patients with COVID-19 in the Bronx, New York. Metabolism 2020;108:154262.

28 Tikellis C, Bernardi S, Burns WC. Angiotensin-converting enzyme 2 is a key modulator of the renin-angiotensin system in cardiovascular and renal disease. Curr Opin Nephrol Hypertens 2011;20:62-8.

29 Fang L, Karakiulakis G, Roth M. Are patients with hypertension and diabetes mellitus at increased risk for COVID-19 infection? Lancet Respir Med 2020;8:e21.

30 Yang G, Tan Z, Zhou L, et al. Effects of angiotensin II receptor blockers and ACE (angiotensin-converting enzyme) inhibitors on virus infection, inflammatory status, and clinical outcomes in patients with COVID-19 and hypertension: a single-center retrospective study. Hypertension 2020;76:51-8.

31 Zhou P, Yang X-L, Wang X-G, et al. A pneumonia outbreak associated with a new coronavirus of probable bat origin. Nature 2020;579:270-3.

32 Hoffmann M, Kleine-Weber H, Schroeder S, et al. SARS-CoV-2 cell entry depends on ACE2 and TMPRSS2 and is blocked by a clinically proven protease inhibitor. Cell 2020;181:271-80.

33 Wang B, Li R, Lu Z, et al. Does comorbidity increase the risk of patients with COVID-19: evidence from meta-analysis. Aging 2020;12:6049-57.

34 Fang X, Li S, Yu H, et al. Epidemiological, comorbidity factors with severity and prognosis of COVID-19: a systematic review and metaanalysis. Aging 2020;12:12493-503. 
35 Zhou Y, He Y, Yang H, et al. Development and validation a nomogram for predicting the risk of severe COVID-19: A multi-center study in Sichuan, China. PLoS One 2020;15:e0233328.

36 Patel U, Malik P, Usman MS, et al. Age-Adjusted risk factors associated with mortality and mechanical ventilation utilization amongst COVID-19 Hospitalizations-a systematic review and metaanalysis. SN Compr Clin Med 2020:1-10.

37 Harrison SL, Fazio-Eynullayeva E, Lane DA, et al. Comorbidities associated with mortality in 31,461 adults with COVID-19 in the United States: a federated electronic medical record analysis. PLoS Med 2020;17:e1003321

38 Lazzaroni MG, Piantoni S, Masneri S, et al. Coagulation dysfunction in COVID-19: the interplay between inflammation, viral infection and the coagulation system. Blood Rev 2021;46:100745.

39 Sathler PC. Hemostatic abnormalities in COVID-19: a guided review. An Acad Bras Cienc 2020;92:e20200834.

40 Kuba K, Imai Y, Rao S, et al. A crucial role of angiotensin converting enzyme 2 (ACE2) in SARS coronavirus-induced lung injury. Nat Med 2005;11:875-9.

41 Oudit GY, Kassiri Z, Patel MP, et al. Angiotensin II-mediated oxidative stress and inflammation mediate the age-dependent cardiomyopathy in ACE2 null mice. Cardiovasc Res 2007;75:29-39.

42 Crackower MA, Sarao R, Oudit GY, et al. Angiotensin-converting enzyme 2 is an essential regulator of heart function. Nature 2002;417:822-8.

43 Liu Z, Huang XR, Chen $\mathrm{H}-\mathrm{Y}$, et al. Loss of angiotensin-converting enzyme 2 enhances TGF- $\beta / S m a d-m e d i a t e d$ renal fibrosis and NF- $\mathrm{BB}$-driven renal inflammation in a mouse model of obstructive nephropathy. Lab Invest 2012;92:650-61.

44 Wu J, Huang J, Zhu G, et al. Elevation of blood glucose level predicts worse outcomes in hospitalized patients with COVID-19: a retrospective cohort study. BMJ Open Diabetes Res Care 2020;8:e001476.

45 Fujii Y, Hirota K. Critical care demand and intensive care supply for patients in Japan with covid-19 at the time of the state of emergency Declaration in April 2020: a descriptive analysis. Medicina 2020;56:530.

46 Xu L, Mao Y, Chen G. Risk factors for 2019 novel coronavirus disease (COVID-19) patients progressing to critical illness: a systematic review and meta-analysis. Aging 2020;12:12410-21.

47 Zhao J, Li X, Gao Y, et al. Risk factors for the exacerbation of patients with 2019 novel coronavirus: a meta-analysis. Int J Med Sci 2020;17:1744-50.

48 Yu C, Lei Q, Li W, et al. Clinical characteristics, associated factors, and predicting COVID-19 mortality risk: a retrospective study in Wuhan, China. Am J Prev Med 2020;59:168-75.

49 Global Health 5050. The COVID-19 Sex-Disaggregated data Tracker, 2020. Available: https://globalhealth5050.org/the-sex-gender-andcovid-19-project/ [Accessed 03 Oct 2020]

50 Gupte M, Thatcher SE, Boustany-Kari CM, et al. Angiotensin converting enzyme 2 contributes to sex differences in the development of obesity hypertension in C57BL/6 mice. Arterioscler Thromb Vasc Biol 2012;32:1392-9.

51 Zhou X, Zhang P, Liang T, et al. Relationship between circulating levels of angiotensin-converting enzyme 2-angiotensin(1-7)-MAS axis and coronary heart disease. Heart Vessels 2020;35:153-61.

52 Liu J, Ji H, Zheng W, et al. Sex differences in renal angiotensin converting enzyme 2 (ACE2) activity are $17 \beta$-oestradiol-dependent and sex chromosome-independent. Biol Sex Differ 2010;1:6.

53 Cook IF. Sexual dimorphism of humoral immunity with human vaccines. Vaccine 2008;26:3551-5.

54 Zheng X-Y, Xu Y-J, Guan W-J, et al. Regional, age and respiratorysecretion-specific prevalence of respiratory viruses associated with asthma exacerbation: a literature review. Arch Virol 2018;163:845-53.

55 Papadopoulos NG, Christodoulou I, Rohde G, et al. Viruses and bacteria in acute asthma exacerbations--a GA2 LEN-DARE systematic review. Allergy 2011;66:458-68.

56 Mahdavinia M, Foster KJ, Jauregui E, et al. Asthma prolongs intubation in COVID-19. J Allergy Clin Immunol Pract 2020;8:2388-91.

57 Johnston SL. Asthma and COVID-19: is asthma a risk factor for severe outcomes? Allergy 2020;75:1543-5

$58 \mathrm{Li}$ X, Xu S, Yu M, et al. Risk factors for severity and mortality in adult COVID-19 inpatients in Wuhan. J Allergy Clin Immunol 2020;146:110-8.
59 Liu S, Zhi Y, Ying S. COVID-19 and asthma: reflection during the pandemic. Clin Rev Allergy Immunol 2020;59:78-88.

60 Ssentongo P, Ssentongo AE, Heilbrunn ES, et al. Association of cardiovascular disease and 10 other pre-existing comorbidities with COVID-19 mortality: a systematic review and meta-analysis. PLoS One 2020;15:e0238215

61 Kimura H, Francisco D, Conway M, et al. Type 2 inflammation modulates ACE2 and TMPRSS2 in airway epithelial cells. J Allergy Clin Immunol 2020;146:80-8.

62 Jackson DJ, Busse WW, Bacharier LB, et al. Association of respiratory allergy, asthma, and expression of the SARS-CoV-2 receptor ACE2. J Allergy Clin Immunol 2020;146:203-6.

63 Nicolau DV, Bafadhel M. Inhaled corticosteroids in virus pandemics: a treatment for COVID-19? Lancet Respir Med 2020;8:846-7.

64 Johnson RM, Vinetz JM. Dexamethasone in the management of covid -19. BMJ 2020;370:m2648.

65 Perez-Guzman PN, Daunt A, Mukherjee S, et al. Clinical characteristics and predictors of outcomes of hospitalized patients with COVID-19 in a multi-ethnic London NHS trust: a retrospective cohort study. Clin Infect Dis 2020:ciaa1091.

66 NCD Risk Factor Collaboration (NCD-RisC). Trends in adult bodymass index in 200 countries from 1975 to 2014: a pooled analysis of 1698 population-based measurement studies with 19.2 million participants. Lancet 2016;387:1377-96.

67 Amoroso A, Magistroni P, Vespasiano F, et al. Hla and ABO polymorphisms may influence SARS-CoV-2 infection and COVID-19 severity. Transplantation 2021;105:193-200.

68 Novelli A, Andreani M, Biancolella M, et al. HLA allele frequencies and susceptibility to COVID-19 in a group of 99 Italian patients. HLA 2020;96:610-4

69 Lorente L, Martín MM, Franco A. Hla genetic polymorphisms and prognosis of patients with COVID-19. Polimorfismos genéticos de Los HLA Y pronóstico de pacientes Con COVID-19. Med Intensiva 2021:45:96-103.

70 Wang W, Zhang W, Zhang J, et al. Distribution of HLA allele frequencies in 82 Chinese individuals with coronavirus disease-2019 (COVID-19). HLA 2020;96:194-6.

71 Barquera R, Collen E, Di D, et al. Binding affinities of 438 HLA proteins to complete proteomes of seven pandemic viruses and distributions of strongest and weakest HLA peptide binders in populations worldwide. HLA 2020;96:277-98.

72 Yamamoto N, Ariumi Y, Nishida N, et al. SARS-CoV-2 infections and COVID-19 mortalities strongly correlate with ACE1 I/D genotype. Gene 2020;758:144944

73 Zeberg H, Pääbo S. The major genetic risk factor for severe COVID-19 is inherited from Neanderthals. Nature 2020;587:610-2.

74 Rhodes JM, Subramanian S, Laird E, et al. Perspective: Vitamin D deficiency and COVID-19 severity - plausibly linked by latitude, ethnicity, impacts on cytokines, ACE2 and thrombosis. J Intern Med 2021;289:97-115.

75 Palacios C, Gonzalez L. Is vitamin D deficiency a major global public health problem? J Steroid Biochem Mol Biol 2014;144 Pt A:138-45.

76 Saito S, Asai Y, Matsunaga N, et al. First and second COVID-19 waves in Japan: a comparison of disease severity and characteristics. J Infect 2021;82:84-123.

77 loannidis JPA, Axfors C, Contopoulos-loannidis DG. Second versus first wave of COVID-19 deaths: shifts in age distribution and in nursing home fatalities. Environ Res 2021;195:110856.

78 Jalali SF, Ghassemzadeh M, Mouodi S, et al. Epidemiologic comparison of the first and second waves of coronavirus disease in Babol, North of Iran. Caspian J Intern Med 2020;11:544-50.

79 Zhao Z, Chen A, Hou W, et al. Prediction model and risk scores of ICU admission and mortality in COVID-19. PLoS One 2020;15:e0236618.

80 Shang Y, Liu T, Wei Y, et al. Scoring systems for predicting mortality for severe patients with COVID-19. EClinicalMedicine 2020;24:100426.

81 Dong $\mathrm{Y}$, Zhou H, Li M, et al. A novel simple scoring model for predicting severity of patients with SARS-CoV-2 infection. Transbound Emerg Dis 2020;67:2823-9.

82 National Institute of Health. Coronavirus disease 2019 (COVID-19) treatment guidelines. Available: https://www.covid19treatmentguid elines.nih.gov/ [Accessed 08 Apr 2021]

83 Wu Z, McGoogan JM. Characteristics of and Important Lessons From the Coronavirus Disease 2019 (COVID-19) Outbreak in China: Summary of a Report of 72314 Cases From the Chinese Center for Disease Control and Prevention. JAMA 2020:323:1239-42. 